\title{
Neurosciences au service de la communication commerciale : manipulation et éthique. Une critique du neuromarketing
}

The Neurosciences in the Service of Marketing Communication: Manipulation and Ethics - A Critique of Neuro-marketing

\section{Didier Courbet et Denis Benoit}

\section{OpenEdition}

\section{Journals}

Édition électronique

URL : http://journals.openedition.org/edc/5091

DOI : 10.4000/edc.5091

ISSN : 2101-0366

\section{Éditeur}

Université Lille-3

\section{Édition imprimée}

Date de publication : 1 juin 2013

Pagination : 27-42

ISBN : 978-2-917562-09-3

ISSN : 1270-6841

\section{Référence électronique}

Didier Courbet et Denis Benoit, « Neurosciences au service de la communication commerciale : manipulation et éthique. Une critique du neuromarketing », Études de communication [En ligne], 40 | 2013, mis en ligne le 01 juin 2013, consulté le 30 avril 2019. URL : http://journals.openedition.org/ edc/5091 ; DOl : 10.4000/edc.5091

Ce document a été généré automatiquement le 30 avril 2019

(c) Tous droits réservés 


\title{
Neurosciences au service de la communication commerciale : manipulation et éthique. Une critique du neuromarketing
}

\author{
The Neurosciences in the Service of Marketing Communication: Manipulation \\ and Ethics - A Critique of Neuro-marketing
}

Didier Courbet et Denis Benoit

1 Récemment apparu, le "neuromarketing " (NM) est un domaine du marketing qui se fonde sur les théories et méthodes des neurosciences - les techniques d'imagerie cérébrale, l'imagerie par résonance magnétique fonctionnelle (IRMf)... - dans le but de mieux comprendre et d'influencer les comportements de consommation et d'achat (voir Droulers \& Roullet, 2010). L'un des principaux domaines du NM concerne les influences des communications commerciales, plus particulièrement les influences non conscientes.

$2 \mathrm{Au}$ sens strict, les neurosciences cognitives étudient le système nerveux central en lien avec les processus psychologiques dits «supérieurs» impliqués dans la perception, la mémorisation, l'intentionnalité, l'évaluation, la planification et l'action. Sur le plan opérationnel, de nombreuses publications présentent le NM comme capable de tirer des enseignements pratiques des neurosciences cognitives pour améliorer les stratégies de communication commerciale et de marketing des organisations (Badoc et Georges, 2010). Le NM se fonde sur le principe selon lequel « mieux on connait le cerveau humain, mieux on peut influencer les comportements d'achat des individus ", notamment à leur insu. Par exemple, Adam Koval, directeur de la société BrightHouse aux États-Unis et que l'on dit précurseur du NM, précise :

La recherche en NM donne un aperçu sans précédent de la pensée du consommateur. Les résultats seront une augmentation des ventes, une préférence pour certaines marques ou encore le fait d'obtenir que les consommateurs se comportent de la façon que l'on désire ${ }^{1}$. 
3 Ainsi voit-on apparaitre depuis quelques années des agences de communication, des instituts d'études ou des départements spécialisés en NM (par exemple les entreprises Neurosense, Neurofocus...). L'utilisation de celui-ci au service des publicitaires soulève d'immanquables questions à la fois théoriques, méthodologiques, stratégiques et éthiques, relatives aux moyens qu'utilise le NM et aux finalités qu'il poursuit. La première partie de notre contribution expliquera qu'au stade actuel des connaissances scientifiques, le NM, au sens strict du terme, c'est-à-dire en tant que domaine qui utilise des techniques d'imagerie cérébrale issues de la recherche en neurosciences n'a pas, à ce jour, d'utilité démontrée pour les stratèges en communication et en marketing: in fine, le NM ne serait-il pas (lui-même) qu'un « coup marketing » ? Et pour quelles raisons ?

4 Nous montrerons, ensuite, que le NM vise bien à influencer à leur insu les personnes en utilisant, en fait, des concepts et méthodes issus de la psychologie scientifique. Sur cette base, et nous fondant sur un recensement des principaux débats éthiques entre les adeptes du NM (les « pro-NM ») et ses détracteurs (les « anti-NM ») nous ouvrirons, dans une seconde partie, une discussion centrée sur la «manipulation des personnes » et l'éthique, relative aux liens entre le NM, la persuasion non consciente et le libre arbitre.

Plus généralement, et à travers une analyse critique de l'utilisation des neurosciences au service de la communication commerciale, nous montrons comment les chercheurs en communication peuvent mettre leurs compétences théoriques pluridisciplinaires, méthodologiques et critiques, au service des acteurs de la communication des organisations. Nous mettons ainsi en évidence l'inutilité fonctionnelle de certaines applications scientifiques « dans l'air du temps » (l'imagerie cérébrale) et l'importance de la réflexion éthique, dans l'intérêt même des organisations. A une époque où il est sans doute capital pour les organisations de se construire une image socialement responsable, empreinte d'éthique (Loneux, 2001), l'utilisation du NM, dont le caractère manipulatoire est socio-médiatiquement dénoncé, pourrait se révéler totalement contre-productif pour les organisations elles-mêmes...

\section{Le neuromarketing : un « coup marketing » à l'utilité douteuse?}

6 Pour montrer que l'imagerie cérébrale n'apporte rien de nouveau ni aux sciences de la communication, ni à la recherche académique en marketing, ni aux professionnels euxmêmes, analysons rapidement deux recherches souvent citées comme significatives par les adeptes du NM.

\section{Deux illustrations montrant que la neuro-imagerie n'apporte rien de nouveau...}

7 Les résultats d'études de produits montrent que lorsque des consommateurs comparent le goût de Coca-cola et de Pepsi-Cola sans savoir quelle marque ils boivent, Pepsi-Cola est généralement préféré à Coca-cola. Le plus souvent, ce résultat est cependant inversé quand les personnes qui testent savent à l'avance quelle marque elles boivent : la bonne image de marque de Coca-Cola influence la préférence déclarée et prend donc le pas sur le goût. McLure S. et al. (2004) ont reproduit ce même test sur des personnes soumises à une analyse par IRM. Lors des tests de goût en aveugle, l'imagerie a révélé une activation 
du putamen ventral proportionnelle au choix : l'activation de cette zone cérébrale est plus forte pour Pepsi. Lorsque le sujet est informé de la marque, une zone cérébrale supplémentaire s'active, le cortex préfontal médian. Cette dernière aire est généralement activée dès que les personnes effectuent des jugements de valeur et des raisonnements. L'excellente image de marque d'un produit, ici Coca-cola, permettrait donc de provoquer dans le cerveau plus de satisfaction que les sensations directement transmises par ses qualités gustatives. Conclusion: si les résultats sont intéressants pour la recherche en neurosciences, ils n'ont manifestement aucune utilité supplémentaire pour les professionnels du marketing!

8 Une autre recherche parue en 2007 a fait grand bruit dans le monde du N. M. Knutson et al . (2007) ont montré que lorsqu'un produit attrayant apparaît sur l'écran, le cerveau du consommateur réagit de manière spécifique : une région du cerveau subcortical, le noyau accumbens (qui est associé à l'anticipation du plaisir) s'active. Quand le prix affiché du produit est excessif, ce qui est un frein à l'achat, c'est une autre zone cérébrale qui s'active : l'insula (zone identifiée comme étant impliquée dans la sensation de douleur). De plus, le cortex pré-frontal lié habituellement au processus de choix est inhibé lorsque le prix est excessif. Il est indéniable que les résultats sont importants pour la recherche scientifique. Cependant, si une retranscription des résultats de l'expérience en termes neurobiologiques semble impressionnante, ils n'ont aucun intérêt pour le professionnel de la communication. En effet, traduisons les résultats en termes communicationnels : "quand on montre un produit attrayant sans voir que le prix est élevé, on a envie d'acheter. Quand on apprend que le prix est élevé, on a une déception et on n'a pas l'intention d'acheter "... Rien de nouveau, donc. Nul besoin d'une IRM pour le savoir, il suffit de le demander aux personnes. Dans ce cas précis, pour connaître les réactions affectives, les raisonnements cognitifs et les intentions d'achat, le langage verbal et le déclaratif sont des indicateurs autant, si ce n'est davantage, valides, plus pratiques et moins chers que l'IRM !

9 Pour les professionnels de la communication et du marketing, en l'état actuel des avancées scientifiques, le NM (au sens strict de domaine du marketing se servant des technologies d'imageries cérébrales des neurosciences) ne fait qu'utiliser métaphores et analogies neurologiques et biologiques pour traduire ce que les spécialistes du comportement du consommateur et de l'influence des médias savent déjà relativement aux pensées, aux affects et aux comportements.

\section{Quatre mythes associés au neuromarketing}

Comme de nombreuses innovations techno-scientifiques, le NM semble associé à quatre types d'imaginaires, à quatre « mythes »:

- le mythe des «nouvelles technologies médicales magiques et toutes puissantes » (l'imagerie par résonance magnétique) ;

- le mythe de « la blouse blanche ", lié à une forte valorisation des rôles des médecins, des neuroscientifiques et de leur crédibilité scientifique ;

- le mythe lié à « l'extraordinaire potentiel de l'organe encore secret et méconnu qu'est le cerveau »; le pourtant très sérieux Pr Gerald Zaltman relaye même l'information selon laquelle « $95 \%$ des activités cognitives du consommateur se déroulent sous le seuil du conscient » $(2004$, p. 12). Un chercheur rigoureux en neurosciences ou en sciences 
cognitives ne se hasarderait pas à une telle quantification!

- le mythe de " la lecture directe des pensées dans le cerveau » grâce à l'imagerie.

11 Rappelons en effet que l'image de l'IRM n'est pas une "photo» mais une «(re)construction» graphique à partir de mesures d'activités neuronales. L'interprétation des images repose en grande partie sur des modèles ou concepts issus de la psychologie cognitive. Or, ces derniers font encore l'objet de discussions (Tiberghien, 2007). Si l'IRM permet des avancées scientifiques majeures dans les sciences neurobiologiques, en permettant par exemple de mieux comprendre certaines fonctions cognitives associées aux structures cérébrales, pour Tiberghien (ibid.), son apport est jusqu'à présent relativement pauvre dans le domaine des sciences humaines. Au niveau théorique, il est fort probable qu'elle n'apportera des connaissances nouvelles sur la cognition, sur les processus de décision et les déterminants des comportements qu'en étant associée à d'autres méthodes de la psychologie. Ces limites de l'IRM valent également pour le domaine de la communication commerciale. En effet, le plus souvent, les applications pratiques basées sur des innovations scientifiques doivent être fondées sur des modèles théoriques scientifiquement valides, notamment sur le plan de la validité prédictive, ce qui est loin d'être le cas aujourd'hui.

\section{De la non-utilité pratique à l'utilité symbolique}

12 Le NM et plus particulièrement l'I.R.M. est-il un bon outil d'aide à la décision pour les professionnels de la communication commerciale? Dans le but de régulièrement améliorer leurs pratiques, ces derniers cherchent :

- des outils d'aide à la décision plus « fins », c'est-à-dire en termes psychométriques, plus sensibles que les outils classiquement utilisés (entretien qualitatif, focus group, questionnaire quantitatif avec notamment des échelles d'évaluation), par exemple, pour évaluer « l'efficacité » des messages ; or, penser que l'IRM permet « d'interroger directement le cerveau » est une erreur ; l'IRM donnant une image de zones cérébrales impliquées dans les tâches que les sujets expérimentaux réalisent, ne met en évidence que des corrélats des activités cognitives, affectives et comportementales des personnes ; - des outils qui permettent de mieux prédire les comportements; si la récente recherche de Falk et al. (2010) tente de développer cette piste, les recherches actuelles utilisant l'IRM n'ont pas de réponse probante à apporter. En psychologie sociale cognitive, il existe d'autres outils plus valides, moins chers et plus faciles à utiliser comme l'analyse des temps de réponses, mesurant notamment l'accessibilité des attitudes et des intentions d'action (Fazio et Towles-Schwen, 1999).

L'IRM, si elle est utile aux neuroscientifiques, n'est donc pas, pratiquement, un outil intéressant pour les stratèges en communication et les hommes de marketing. Dès lors, pourquoi agences de communication, instituts d'études et annonceurs s'intéressent-ils au NM ? Nous émettons une double hypothèse. Premièrement, le NM, sous une apparence scientifique, permettrait de vendre nombre de nouvelles prestations et outils à des acteurs professionnels qui n'ont pas les connaissances théoriques et méthodologiques pour en évaluer la validité. Deuxièmement, mettre un peu de discours neuroscientifique, à la pointe du progrès, dans son discours quotidien pourrait contribuer à enrichir l'image de l'organisation et accroitre la crédibilité perçue. Cela lui donnerait un avantage sur ses concurrents lui permettant de mieux se positionner sur les marchés de ventes interorganisations hyper-concurrentiels. 
14 Si l'utilité pratique du neuromarketing en tant qu'outil d'aide à la décision et à l'amélioration de l'efficacité des pratiques de communication nous semble nulle, son utilité symbolique jouant sur les représentations sociales (l'image de marque) de l'organisation, pourrait être bien réelle. Cependant comme nous le montrons plus loin, l'utilisation du NM pourrait au final se révéler contre-productive car en contradiction avec les valeurs sociales ambiantes.

\section{Derrière le neuromarketing, une certaine application de la psychologie expérimentale}

15 Si un premier principe du NM lié à l'utilité pratique de l'IRM est invalidé, un deuxième principe doit être analysé : la communication commerciale peut-elle vraiment influencer sans que les personnes en soient conscientes?

16 En examinant de plus près les recherches qui n'ont pas utilisé l'IRM et qui sont tout de même présentées comme faisant partie du NM (voir Zaltman, 2004), on s'aperçoit qu'il y a davantage de recherches mobilisant des concepts de la psychologie cognitive, de la psychologie sociale et de la psychologie des émotions que du champ des neurosciences. Les concepts et méthodes proviennent majoritairement des domaines scientifiques qui ne font pas appel au substrat neurologique comme la cognition implicite, la mémoire implicite, les automatismes cognitifs et leur bagage méthodologique (eye-tracking, mesure des temps de réponse, mesures psychophysiologiques...). Ainsi, dans la mesure où les recherches de référence sont dans leur grande majorité plus proches des «sciences de l'esprit » que des « sciences du cerveau », le terme «NM », en fait non adapté, est tout de même utilisé en raison de l'imaginaire social valorisé qui lui serait associé.

$17 \mathrm{Au}$ cours des deux dernières décennies, de nombreux travaux ont effectivement permis de montrer et de mieux comprendre les processus non conscients impliqués dans la réception et l'influence des communications commerciales, soit dans un but de recherche fondamentale sans visée applicative (par exemple, en sciences de l'information et de la communication, Courbet, Vanhuele et Lavigne, 2008), soit de recherche appliquée (par exemple Nordhielm, 2002). Ce dernier auteur a montré qu'un message publicitaire simple, court et répété, reçu en condition de faible implication, c'est-à-dire lorsque le récepteur n'est pas fortement intéressé par la publicité, rend plus fluide les réseaux cognitifs associés à la perception et à la mémorisation de la marque. Les consommateurs n'ont pas conscience de la formation de cet effet de « simple exposition » (mere exposure), rendant la marque plus familière et conduisant à davantage l'apprécier. Lorsque le taux de répétition de la publicité augmente, ce type de message ne conduit pas à un effet de lassitude chez le récepteur en raison de sa faible implication et du faible niveau de ressources cognitives qu'il alloue au message.

18 Au niveau pratique, certaines agences de communication ont intégré ce dernier résultat dans la conception de leurs plans médias. Les publicitaires savent alors qu'ils peuvent aisément augmenter les taux de répétition pour les produits peu impliquant, les effets bénéfiques à la marque augmenteront proportionnellement.

19 Certaines recherches s'intéressent également à l'affectif puisqu'on sait qu'une marque vue dans un état émotionnel positif bénéficie d'un transfert affectif. Cet effet d'assimilation est sans doute automatique, c'est-à-dire réalisé sans conscience et très rapidement en mémoire (Meyers-Levy et Sternthal, 1993). Les stratèges en 
communication savent qu'il vaut mieux parrainer des événements ou des programmes de télévision générant des émotions positives que des événements générant des émotions négatives.

\section{Ethique du neuromarketing : libre arbitre et manipulation}

Comme une grande partie des pratiques de NM - ou définies comme appartenant à ce domaine... - visent à influencer les personnes sans qu'elles en aient conscience, de nombreux débats éthiques ont lieu entre pro- et anti-NM concernant son caractère manipulatoire. Pour des raisons de place, nous présentons une synthèse des huit principaux thèmes de débats sous forme de tableau.

Tableau : Les huit principaux thèmes de débats éthiques entre pro et anti-neuromarketing

\begin{tabular}{|c|c|c|}
\hline $\begin{array}{ll}\text { Les } & \text { huit } \\
\text { principaux } & \\
\text { thèmes } & \end{array}$ & $\begin{array}{l}\text { Arguments des «anti- } \\
\text { neuromarketing } "\end{array}$ & Arguments des «pro-neuromarketing » \\
\hline $\begin{array}{l}\text { Thème } 1 \\
\text { La nature } \\
\text { l'outil }\end{array}$ & $\begin{array}{l}\text { Le NM est un puissant outil de } \\
\text { "manipulation». Il réactive le } \\
\text { mythe de «l'influence non } \\
\text { consciente par les images } \\
\text { subliminales». }\end{array}$ & $\begin{array}{l}\text { Le NM n'est qu'une mesure } \\
\text { supplémentaire de la qualité des messages } \\
\text { publicitaires et des actions de marketing. } \\
\text { Il n'existe aucune « zone de l'achat dans le } \\
\text { cerveau " qu'il suffirait de stimuler pour } \\
\text { faire automatiquement acheter. }\end{array}$ \\
\hline $\begin{array}{l}\text { Thème } 2 \\
\text { Le NM et la santé } \\
\text { publique }\end{array}$ & $\begin{array}{l}\text { Le NM peut faire augmenter les } \\
\text { " épidémies " causées par les } \\
\text { excès du marketing: obésité, } \\
\text { diabète, addiction ou jeux } \\
\text { d'argent, addiction au tabac ou } \\
\text { à l'alcool, désordres } \\
\text { alimentaires. }\end{array}$ & $\begin{array}{l}\text { Sur ce thème, c'est le procès du } \\
\text { capitalisme et de la société " de } \\
\text { consommation » actuelle qu'il faut faire. Il } \\
\text { ne faut pas confondre l'outil (le NM) et la } \\
\text { finalité des actions qui sont } \\
\text { fondamentalement économiques. }\end{array}$ \\
\hline $\begin{array}{l}\text { Thème } 3 \\
\text { Les outils d'études } \\
\text { utilisés par le NM }\end{array}$ & $\begin{array}{l}\text { Les médecins qui font des } \\
\text { études en NM violent le } \\
\text { serment d'Hippocrate, car ils } \\
\text { sont censés aider les individus } \\
\text { et la société. }\end{array}$ & $\begin{array}{l}\text { Bien utilisés, les outils du NM ne font pas } \\
\text { de mal aux êtres humains; s'il y a } \\
\text { problème, il est causé par une erreur } \\
\text { humaine ponctuelle. Il faut expliciter et } \\
\text { appliquer une charte très stricte de «bon } \\
\text { fonctionnement» avec les médecins. En } \\
\text { demandant l'aval aux comités éthiques } \\
\text { avant études, il n'y a pas de problème } \\
\text { éthique. }\end{array}$ \\
\hline $\begin{array}{l}\text { Thème } 4 \\
\text { Le NM et les } \\
\text { enfants }\end{array}$ & $\begin{array}{l}\text { Le NM contribue à la } \\
\text { manipulation publicitaire } \\
\text { d'enfants (faibles défenses } \\
\text { cognitives). }\end{array}$ & $\begin{array}{l}\text { Le NM donne la possibilité de mieux } \\
\text { adapter les messages publicitaires au } \\
\text { cerveau des enfants, pour mieux les } \\
\text { respecter. }\end{array}$ \\
\hline
\end{tabular}




\begin{tabular}{|c|c|c|}
\hline $\begin{array}{l}\text { Thème } 5 \\
\text { Le NM et les } \\
\text { achats inutiles }\end{array}$ & $\begin{array}{l}\text { Le NM conduit à faire acheter } \\
\text { des marques et des produits } \\
\text { dont les adultes et les } \\
\text { adolescents n'ont pas besoin, } \\
\text { qui leur sont inutiles. }\end{array}$ & $\begin{array}{l}\text { Les adultes et adolescents ont besoin de } \\
\text { produits et marques pour s'intégrer au } \\
\text { groupe, construire leur identité, c'est un } \\
\text { «rite de passage" pour une bonne } \\
\text { insertion sociale. }\end{array}$ \\
\hline $\begin{array}{l}\text { Thème } 6 \\
\text { Transparence des } \\
\text { pratiques } \\
\text { publicitaires } \\
\text { utilisant le NM }\end{array}$ & $\begin{array}{l}\text { Les organisations qui utilisent } \\
\text { le NM se cachent; les } \\
\text { consommateurs sont en droit } \\
\text { de savoir s'ils sont manipulés } \\
\text { ou non. }\end{array}$ & $\begin{array}{l}\text { C'est à cause des détracteurs qui accusent, } \\
\text { à tort, le NM que les organisations qui le } \\
\text { pratiquent se cachent. }\end{array}$ \\
\hline $\begin{array}{l}\text { Thème } 7 \\
\text { Le NM et la } \\
\text { réglementation }\end{array}$ & $\begin{array}{l}\text { Les pratiques peuvent dériver } \\
\text { vers de graves excès. Il faut que } \\
\text { le législateur réglemente, pas } \\
\text { uniquement en France (1) mais } \\
\text { dans tous les pays. }\end{array}$ & $\begin{array}{l}\text { Pas besoin de réglementation publique. Il } \\
\text { y a une auto-réglementation par la } \\
\text { profession qui a déjà ses propres règles } \\
\text { éthiques. Les professionnels sont des gens } \\
\text { responsables qui peuvent davantage } \\
\text { expliciter ou renforcer leur auto } \\
\text { réglementation. }\end{array}$ \\
\hline $\begin{array}{l}\text { Thème } 8 \\
\text { Le NM et la liberté } \\
\text { individuelle }\end{array}$ & $\begin{array}{l}\text { Le NM est contraire au principe } \\
\text { démocratique de libre arbitre } \\
\text { des individus, de libre choix et } \\
\text { contraire à la liberté. }\end{array}$ & $\begin{array}{l}\text { Le NM n'entrave pas la } \\
\text { liberté individuelle : l'individu a toujours } \\
\text { le choix de son achat. Si le produit est } \\
\text { mauvais, le ré-achat ne se fera pas. La } \\
\text { persuasion est partout dans nos sociétés. } \\
\text { La communication commerciale a } \\
\text { toujours utilisé les outils des sciences } \\
\text { humaines (ex : psychanalyse) pour mieux } \\
\text { influencer. }\end{array}$ \\
\hline
\end{tabular}

Notes-Tableau conçu à partir d'une revue de littérature et des quarante sites internet apparaissant aux quarante premières positions sur le moteur de recherche Google, le 15 mai 2012, à partir des mots clés « neuromarketing; éthique ». (1) Selon la loi de bioéthique du 7 juillet 2011, art. 45, «les techniques d'imagerie cérébrale ne peuvent être employées qu'à des fins médicales ou de recherche scientifique, ou dans le cadre d'expertises judiciaires».

21 Nous constatons dans ce tableau que la notion de «manipulation » figure au centre des débats. Celle-ci est le plus ordinairement définie comme l'utilisation délibérée de moyens de communication dans le but d'abuser du libre arbitre de la personne, à son insu et avec un objectif intéressé (voir Beauvois, 2011 ; Benoit, 2006, 2012). Dès lors, la communication commerciale qui emploie les procédés que s'attribue le NM ne réduirait-elle pas de facto, et dans des proportions définitivement inacceptables sur un plan éthique, la liberté de choix des individus?

\section{Le neuromarketing : une atteinte à la liberté rationnelle}

Le NM - ou plutôt ce que l'on range sous cette dénomination - cherchant à influencer, sans que les personnes en aient conscience, les croyances et désirs en amont de l'action d'achat, renvoie donc à un ensemble de contraintes limitatives de la liberté rationnelle. Celle-ci est considérée, au sens de Descartes, comme l'état de la personne qui juge et agit en pleine conscience. Or, un enseignement majeur des psychologies cognitive et sociale 
est de montrer que notre "machine à penser ", c'est-à-dire notre cerveau, ne nous permet pas toujours de savoir comment nous fonctionnons dans notre vie sociale : il ne nous donne pas la possibilité d'être conscient d'un grand nombre des mécanismes fondamentaux nous conduisant à produire de la signification, à former nos connaissances, nos croyances, nos idées, nos affects, nos actions... (voir Beauvois, 2010). Cette impénétrabilité psychologique, cognitive et émotionnelle, nous empêche assez souvent d'avoir conscience des véritables déterminants de nos actions (Wegner, 2002; Proust, 2005). Bien documenté sur cette question, le NM cherche à exploiter cette limite du cerveau humain, ce qui peut conduire la personne à ressentir, à tort, un sentiment d'autodétermination l'amenant à penser qu'elle a acheté en toute liberté. En outre, l'homme, ressentant le besoin de donner à lui-même et aux autres des justifications à ses croyances, à ses désirs ou à ses actions, construit la signification de ses pensées et de ses actes en leur attribuant souvent, sans en avoir conscience et a posteriori, de «fausses raisons » ou «fausses causes ». Si l'individu achète un produit à la suite d'influences non conscientes de la communication commerciale, il ressentira souvent un besoin irrépressible de justifier rationnellement son achat à ses propres yeux ou aux yeux des autres.

De nombreux manuels publicitaires, dans une perspective de NM, expliquent que, pour augmenter la probabilité d'achat, le publicitaire doit inclure dans les messages, en parallèle des techniques incitant à déclencher des influences non conscientes - tel le conditionnement évaluatif provoqué par l'association d'une marque avec un stimulus chargé affectivement de façon positive (un animal attendrissant, une belle femme...) - des arguments rationnels et socialement acceptables. Même si ces derniers n'ont pas de lien direct avec les véritables causes de l'achat, ils permettent à l'acheteur, a posteriori, d'une part, de retrouver une consonance cognitive et de se rassurer psychologiquement et, d'autre part, de donner aux autres une image de soi socialement acceptable.

\section{Un appel aux débats publics et à l'éthique}

24 En utilisant délibérément des procédés d'influence non consciente, le «NM » cherche donc à réduire la liberté de choix des personnes dans le domaine de la consommation. Or, de telles atteintes ne peuvent évidemment pas ne pas faire réagir. Ainsi, indiquant que les comportements humains sociaux ne peuvent s'expliquer seulement par les processus neuronaux, Dehaene (2009) s'interroge-t-il à propos de "l'acide matérialiste des neurosciences [...] qui rongerait [...] les fondements même de notre société, en fragilisant voire en anéantissant ses piliers que sont le libre arbitre, la responsabilité, l'identité individuelle et le jugement moral ». Dans un même sens, des chercheurs et universitaires appellent, depuis plusieurs années, à des débats sur l'éthique de ces pratiques. Par exemple, dans LeMonde.fr du 26 juin 2012, un collectif de chercheurs autour de Guillaume Dumas demande « un débat le plus large possible sur la présence de la publicité [visant à activer les automatismes cognitifs] dans l'espace public», la liberté d'expression ne devant pas aller «sans une autre liberté complémentaire de la première: la liberté de non-réception ", chaque citoyen devant in fine pouvoir " choisir où et quand il souhaite accéder à de l'information publicitaire ».

Des chercheurs adeptes du NM se lancent également sur la voie de l'éthique, à un niveau plus « technique » cependant. Ainsi, Roullet et Droulers (2012) insistent sur la «nécessité grandissante pour la neuroscience du consommateur académique et le NM de [...] 
concevoir des règles éthiques ou déontologiques, acceptées et appliquées par tous, permettant une plus grande transparence et traçabilité au bénéfice des clientsentreprises, des consommateurs et des pouvoirs publics de régulation ». Par ailleurs, alors que les organisations cherchent à se construire une image "socialement responsable et éthique » (Loneux, 2001), nombre d'enquêtes journalistiques², relayées par les médias sociaux, dénoncent les contradictions entre les discours à base d'arguments éthiques des organisations et leurs pratiques effectives. Dès lors, l'utilisation du NM pourrait se révéler contre-productive pour les organisations et contraire aux valeurs sociales ambiantes.

\section{Vers une éthique de responsabilité}

Selon nous, les techniques que s'attribue le NM - à condition bien entendu qu'elles fassent (en permanence) la preuve de leur validité et de leur efficacité - peuvent représenter, comme la langue d'Esope, la meilleure et la pire des choses. Tout dépend, évidemment et comme toujours, de ce que l'on en fait... Dans le champ de la communication commerciale (au moins), ce n'est probablement pas une conception «kantienne » de l'éthique et de la morale - un «impératif catégorique » du type : «il ne faut jamais manipuler» (voir Benoit, 2012) - qui est la plus pertinente et qui doit s'appliquer. Car, dans notre type de société, souvent dite péjorativement «de consommation ", peut-on raisonnablement reprocher à la communication publicitaire (au sens large), dont c'est le rôle et l'objectif patent et manifeste, de faire " au mieux » pour vanter les produits qu'elle prend en charge? D'autant que la frontière entre la simple «influence» (vue habituellement comme acceptable) et la «manipulation» (ordinairement considérée comme intolérable) est sans doute impossible à tracer d'une façon parfaite et définitive. En l'occurrence, ce n'est pas tant le procès de la publicité et de ses méthodes qu'il s'agit d'instruire, que de recalibrer politiquement et idéologiquement nos attentes sociales et sociétales.

Dès lors, laissons plutôt de côté les questions spécifiquement déontologiques liées à la communication commerciale pour seulement envisager (d'une façon qui ne peut être, ici, qu'extrêmement succincte) ses aspects proprement éthiques. Nous dirons alors que c'est une " éthique de responsabilité », celle qui cherche à toujours "faire la part des choses ", qui « doit » sans doute - à l'inverse de celle de règles déontologiques, l'application de principes éthiques ne peut jamais se commander... - être mise en œuvre. Car, comme l'affirme Weber (1959: 207) :

"Pour atteindre des fins 'bonnes', nous sommes la plupart du temps obligés de compter avec, d'une part, des moyens malhonnêtes ou pour le moins dangereux, et d'autre part, la possibilité ou encore l'éventualité de conséquences fâcheuses ».

Une éthique de responsabilité qui, par-delà la complexité des débats axiologiques sur la neutralité, l'amoralité (Todorov, 1983) des moyens et des techniques ${ }^{3}$, nous incite à considérer la technique comme pouvant aussi bien être utilisée pour « asservir » que pour « libérer ». Pour asservir, lorsqu'il est question, "profanant le sanctuaire de la pensée intime», d'attenter massivement et d'une façon inconsidérée à «l'autonomie des consommateurs", (voir Murphy et al., 2008). Pour "libérer", lorsqu'il est question d'améliorer la façon dont les organisations créent des produits et en font la publicité afin qu'ils deviennent plus utiles fonctionnellement pour les consommateurs, moins délétères pour la santé publique ou plus respectueux de l'environnement. 


\section{En guise de conclusion}

29 A travers une analyse critique de l'utilisation des neurosciences au service de la communication commerciale, nous avons montré comment les chercheurs en communication peuvent mettre leurs compétences théoriques pluridisciplinaires, méthodologiques et critiques, au service des acteurs de la communication des organisations.

$30 \mathrm{Si}$, plus généralement, communication et manipulation sont, dans les faits, difficilement dissociables, il revient également aux chercheurs et penseurs travaillant dans le domaine des sciences de l'information et de la communication, et plus généralement des sciences humaines et sociales, d'interroger les bases éthiques et idéologiques d'un système sociocommunicationnel, impliquant des organisations, qui, en utilisant- voire, en détournant - la science, cherchent systématiquement à faire davantage pencher le balancier du côté de la manipulation (Beauvois, 2011). De fait, l'éthique «de responsabilité » que nous préconisons, à l'inverse de l'éthique dite «de conviction »voir Hottois (1996), qui définit les deux éthiques l'une par rapport à l'autre - implique une position située en permanence «sur le fil du rasoir ». En effet, sur le plan éthique, il ne peut être question d'obéir à de grands principes définitivement intransgressibles mais, au contraire, d'évaluer les comportements en fonction de leurs conséquences pratiques, par définition toujours problématiques. Toutefois, lorsque les intérêts économiques prennent l'importance cruciale qu'ils occupent dans notre type de société, faire confiance aux seuls raisonnements éthiques/moraux des organisations marchandes est sans doute insuffisant. Le législateur a également un rôle à jouer. Informé au mieux par les chercheurs et les praticiens au fil de l'évolution des nouvelles connaissances - et accompagné sur le plan déontologique par les institutions et organismes ad hoc - il doit imposer des règles formelles et claires.

31 Il peut être attribué un autre rôle aux chercheurs motivés par des idéologies humanistes, celui d'expliquer aux consommateurs quels procédés de communication/manipulation sont utilisés par les publicitaires et comment ils agissent sur eux sans qu'ils en aient conscience. Savoir comment fonctionnent ces procédés permet, dans certains cas, d'en atténuer les effets non conscients et de prendre des décisions d'achat de manière plus éclairée, à partir de critères plus « rationnels ».

\section{BIBLIOGRAPHIE}

Badoc M. et Georges P., (2010), Le neuromarketing en action : parler et vendre au cerveau, Paris, Eyrolles, $336 \mathrm{p}$.

Beauvois J.-L., (2010), Préface, in Courbet D. (éd.), Objectiver l'Humain ? Volume 2 : Communication et expérimentation, Paris, Éditions Hermès Lavoisier, pp. 17-24. 
Beauvois J. L., (2011), Les Influences sournoises : précis des manipulations ordinaires, Paris, Bourin, $364 p$.

Benoit D. (dir.), (2012), Ethique et communication, Sarrebruck, Éditions Universitaires Européennes, $236 \mathrm{p}$.

Benoit D., (2006), Une éthique de la communication : la requête (ou revendication) impossible..., in Market Management, 4 (« Entre Manipulation et éthique »), Éditions Eska, pp. 37-53.

Courbet D., Vanhuele M. et Lavigne F., (2008), Les effets persuasifs de l'e-publicité perçue sans conscience en vision périphérique. Implications pour les recherches sur la réception des médias, in Questions de Communication, 14, pp. 197-219.

Dehaene S., (2009), La neuroéthique, une nouvelle frontière pour les sciences humaines, in La Vie des idées, 6 octobre 2009. URL : http://www. laviedesidees.fr/La-neuroethique-une-nouvelle.html consulté le 20 juillet 2012 .

Droulers O. et Roullet B., (2010), Neuromarketing. Le marketing revisité par les neurosciences du consommateur?, Paris, Dunod, 224 p.

Droulers O. et Roullet B., (2012), Ethique et Déontologie du Neuromarketing, $28^{\mathrm{e}}$ Congrès de l'Association Française de Marketing, Brest, 9-11 mai 2012.

Falk E. B., Berkman E. T., Mann T., Harrison B. et Lieberman M. D., (2010), Predicting Persuasion-Induced Behavior Change from the Brain, in The Journal of Neuroscience, 30(25), pp. 8421-8424.

Fazio R. H. et Towles-Schwen T., (1999), The MODE Model of Attitude-Behavior Process, in Chaiken S. et Tropes Y. (éds.), Dual-Process Theories in Social Psychology, New York, The Guilford Press, pp. 97-116.

Hottois G., (1996), Éthique de la responsabilité et éthique de la conviction, Laval théologique et philosophique, 52, 2 juin 1996, pp. 489-498. URL : http://id.erudit.org/iderudit/401006ar consulté le 19 août 2012.

Knutson B. et al., (2007), Neural Predictors of Purchases, in Neuron, 53, 1 January, pp. 147-156.

Loneux C., (2001), Proclamation d'une nouvelle matérialité pour l'éthique dans les entreprises - forme d'organisation inédite du travail ou réaménagement des effets de la communication symbolique?, in Communication et organisation [En ligne], 19, URL : http:// communicationorganisation.revues.org/2493 consulté le 16 janvier 2011.

Mc Clure S. et al., (2004), Neural correlates of behavioral preference for culturally familiar drinks, in Neuron, 44, October, pp. 379-387.

Meyers-Levy J. et Sternthal P., (1993), A Two-Factor Explanation of Assimilation and Contrast Effects, in Journal of Marketing Research, 30, pp. 359-368.

Murphy E. R., Liles J. et Reiner P. B., (2008), Neuroethics of neuromarketing, in Journal of Consumer Behaviour, 7, pp. 293-302.

Nordhielm C. L., (2002), The influence of level of processing on advertising effects, in Journal of Consumer Research, 29 (December), pp. 371-382.

Proust J., (2005), La nature de la volonté, Paris, Gallimard, 363 p.

Tiberghien G., (2007), Entre neurosciences et neurophilosophie : la psychologie cognitive et les sciences cognitives, in Psychologie Française, 52 (3), pp. 279-297. 
Todorov T., (1983), Eloquence, morale et vérité, in Les manipulations, Le Genre Humain 6, Paris, Éditions Complexe, pp. 26-41.

Weber M., (1959), Le savant et le politique, Paris, Plon, 221 p.

Wegner D., (2002), The illusion of the conscious will, Cambridge, MIT Press, 419 p.

Zaltman G., (2004), Dans la tête du client, ce que les neurosciences disent au marketing, Paris, Éditions d'Organisation, $381 \mathrm{p}$.

\section{NOTES}

1. www.journaldunet.com/management/dossiers/0702175-neuromarketing/controverse/2.shtml consulté le 20 août 2012.

2. Voir l'émission TV: Neuromarketing - Votre cerveau les intéresse, http://www.youtube.com/ watch?v=ewsGsoixGj4 consulté le 16 janvier 2012.

3. Ce débat, récurrent et complexe, que nous ne pouvons développer dans cet article faute de place, a notamment été alimenté, dans divers domaines disciplinaires, par des auteurs comme Dagognet, Debray, Dupuy, Ellul, Habermas, Heidegger, Jonas, Marcuse, Marx, Simondon, Stiegler, Weber...

\section{RÉSUMÉS}

Le neuromarketing prétend être capable de tirer des enseignements des neurosciences pour influencer les comportements d'achat des personnes, notamment à leur insu, et permettre l'amélioration des stratégies de communication commerciale. Nous défendons une autre hypothèse : le neuromarketing serait essentiellement un «coup marketing ». A travers une analyse critique de cette instrumentalisation des neurosciences, nous montrons comment les chercheurs en communication peuvent employer leurs compétences au service des organisations en mettant en évidence l'inutilité de certaines applications scientifiques "dans l'air du temps » et l'importance d'une réflexion éthique dans l'intérêt même des organisations.

By using knowledge drawn from the neurosciences, neuro-marketing claims to be capable of influencing the purchasing behavior of individuals without their being conscious of it. In this sense, neuro-marketing could be seen as essentially a marketing trick. Through a critical analysis of this instrumentalization of the neurosciences, we show how communication researchers can use their skills in the service of organizations by highlighting the uselessness of certain fashionable scientific applications and the importance of an ethical reflection in the interest of organizations. 
INDEX

Mots-clés : neuromarketing, communication commerciale, persuasion, éthique, manipulation, déontologie

Keywords : neuromarketing, marketing communication, persuasion, ethics, manipulation, deontology

\section{AUTEURS}

\section{DIDIER COURBET}

IRSIC - Aix-Marseille Université

Didier Courbet, Directeur adjoint de l'Institut de Recherche en Sciences de l'Information et la Communication (IRSIC), est Professeur en sciences de l'information et de la communication à Aix-Marseille Université. Il travaille sur la réception et l'influence des médias, notamment sur les processus d'influence non conscients de la communication commerciale. Publications récentes : «The prescriptive power of the television host. A transposition of Milgram's obedience paradigm to the context of TV game show ", European Review of Applied Psychology, 62, 2012 (avec J. L. Beauvois et D. Oberlé) et Objectiver l'Humain? Vol. 2 : Communication et expérimentation, Hermes Lavoisier, 2010. Adresse électronique : didier.courbet@orange.fr.

\section{DENIS BENOIT}

LERASS-CERIC - Université Montpellier 3

Denis Benoit, membre de l'équipe CERIC du laboratoire pluridisciplinaire d'études et de recherches appliquées en sciences sociales (LERASS), est Professeur en sciences de l'information et de la communication à l'Université Paul Valery - Montpellier 3. Ses recherches portent sur le domaine de l'information-communication envisagé selon un point de vue qui tend à imbriquer, d'une façon indissociable, les questions théoriques, pratiques, techniques aux questions éthiques. Dernière publication : Information-Communication : théories - pratiques - éthique - De la psychothérapie aux techniques de vente (Editions ESKA, 2012). Il co-dirige la revue Communication \& Management. Adresse électronique : denis.benoit.univ@gmail.com. 